\title{
Analysis of Market Equilibrium Based on Co-evolution in Electricity Spot Market
}

\author{
Guosheng Hu${ }^{1}$, Hechun Wang ${ }^{2}$, Kun Fan ${ }^{2}$, Haoyong Chen ${ }^{3, *}$, Shengsheng Deng ${ }^{3}$ and Shengzhi Duan ${ }^{3}$ \\ ${ }^{1}$ State Grid Jiangxi Electric Power Co., LTD., Nanchang 330077, Jiangxi Province, China \\ ${ }^{2}$ Jiangxi Electric Power Trading Centre Co., LTD, Nanchang 330077, Jiangxi Province, China \\ ${ }^{3}$ School of Electric Power, South China University of Technology, Guangzhou 510641, Guangdong Province, China
}

\begin{abstract}
By solving the Nash equilibrium of the electricity market, it is possible to observe the game process of market entities under different boundary conditions and predict the future trend of the market. In order to study the state of market equilibrium in the power spot market, firstly we constructed a bi-level equilibrium model. The upper layer is the problem of maximizing the profit of power generation enterprises under the bidding constraint, and the lower layer is the security constraint economic dispatch with the goal of maximizing social welfare. The traditional solution transforms the bi-level model into MPEC or EPEC through optimal conditions, but they are generally non-convex and difficult to solve. In this regard, the coevolution algorithm is used to solve the bi-level model, and it is proved that the result of co-evolution under a limited strategy set is equivalent to the Nash equilibrium. Finally, an example of PJM 5 machine with 5 nodes is used to analyse the power market equilibrium in the spot market.
\end{abstract}

\section{Introduction}

The electricity market equilibrium is a stable state reached after the dynamic evolution of the market between the supply and demand sides of the bidding game. By solving the equilibrium point of the electricity market, it is possible to observe the decision-making evolution process of the market entities, and also to predict and analyse the Nash equilibrium point under different market boundary conditions. In this way, suggestion for improvement of market rules and design can be come up with.

The game between market entities, and the process of information feedback through the clearance of the electricity market can be modelled as a bi-level model [1] [2]. In the method of solving the market equilibrium point, the classic equilibrium analysis method believes that the game party is completely rational and finds the equilibrium solution of the game under the premise of sufficient information (Cournot model) [3]. [4] solves the market equilibrium point by constructing an bi-level equilibrium model. The upper level is the profit maximization problem of power generation enterprises, and the lower level is the optimal flow problem of social welfare maximization. By replacing the lower-level problem with the first-order optimal conditions $[5,6]$, the bi-level model is converted into a single-layer model [7], namely mathematical program with equilibrium constraints [8] (MPEC). Furthermore, an equilibrium program with equilibrium constraints (EPEC) is formed by combining the MPEC model of the optimal decisionmaking of multiple market entities to solve the equilibrium of the entire market. [9] discussed the market equilibrium of the natural gas and electric energy coupling market through EPEC. [10] studied the influence of market power and market power mitigation policies on market equilibrium through the MPEC model.[11] studied the impact of green certificates on market equilibrium in an oligopoly market through EPEC.

The above study of market equilibrium using the classic game theory method belongs to the research of static game, but this can only be applied to the mature stage of market development. In the electricity market, the cost function of participants and their competition information are generally confidential. Besides, the condition of complete rationality is difficult to satisfy, therefore, the use of traditional equilibrium theory to model the electricity market may cause problems. In addition, the mathematical process of MPEC and EPEC models is cumbersome and complex, and it is prone to be non-convex or difficult to solve when dealing with mathematical models with high-dimensional slack variables and complex constraints. Therefore, some scholars proposed to use heuristic algorithm [12] such as co-evolution algorithm [13] to solve the bi-level model. Co-evolutionary algorithms have been widely used since they were proposed, including solving power system reactive power optimization [14,15], robust scheduling [16] and market equilibrium [17]. Literature [18] uses coevolutionary algorithm to find the pure strategy equilibrium in the market, and proves that the algorithm has strong ability to search the Nash equilibrium [19].

In the current modelling of the electricity market, there are few considerations of multi-block bidding and safety

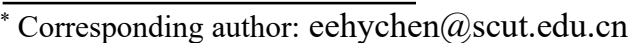


constraints. In this regard, this article first constructed an upper and lower two-level equilibrium model of multiblock bidding for power generation companies and safeconstrained economic dispatch of power dispatch agencies in a spot environment, then uses a co-evolution algorithm to solve the market equilibrium point, and finally analyses the market equilibrium point in the spot environment Features.

\section{Market equilibrium model}

\subsection{Market rules}

In this article, the units are distributed on various nodes of the power network, and participate in the day-ahead market through bidding in the power trading centre. In the day-ahead market, units declare their willingness to trade capacity and price of power, and the declaration can be divided into multiple stages to reduce the risk of failure. The declared information will take effect within 24 hours of the next day and no modification of the declared information is allowed within the day. At the same time, in order to avoid the unit holding the remaining power generation capacity to manipulate the market, the sum of the bidding capacity should be the upper limit of the unit's bidding capacity.

For the demand sides, in order to ensure sufficient power supply, the marginal utility and the demand of electricity is declared at the same time, and the sum of the winning bidding electricity of all users at the same node is equal to the predicted load of the node.

After the power dispatching agency receives the declaration information from each market entity, it conducts a day-ahead market clearance program once a day. Market clearing uses the DC power flow model to construct network security constraints. The objective function of clearing is a social welfare maximization model that comprehensively considers the supply-side and demand-side declarations. The result of clearing is the amount of electricity purchased or sold by market entities per hour, and the system marginal price.

In the bidding declaration stage, all market entities make decisions with the goal of maximizing their own profits. After multiple rounds of bidding, the market will reach an equilibrium state, that is, no market subject can unilaterally change its strategy to increase profits. Since the best response strategy of market entities is related to the market clearing conditions shared by all market entities, it is a multi-leader-common-follower game problem.

This paper constructs a bi-level model to describe the bidding strategy behaviour and process of market entities [20]. The upper-level model is an optimization model for maximizing the profits of power generation companies, with the curve of bidding declaration as the optimization variable, and the restriction of bidding declaration in market rules as constraints. The lower-level model aims to describe the process of market clearing, with the maximization of social welfare as the objective function, and the constraints include unit operation constraints, network security constraints, and market rules constraints.
The upper and lower models are interconnected and restricted. The optimal decision-making process of the power generation company in the upper model depends on the lower node electricity price information and cleared bidding capacity, while the basic data for clearing of the lower market is the declaration generated by the optimal decision of the upper model curve. Details as follows:

\subsection{Upper model}

$$
\begin{gathered}
\min _{\alpha_{i, j}, P_{i, j}^{\mathrm{Gmax}}} \sum_{t} \sum_{i \in \Psi_{n}} \sum_{j}\left(-\lambda_{n}(t) P_{i, j}^{\mathrm{G}}(t)+\lambda_{i, j}^{G} P_{i, j}^{\mathrm{G}}(t)\right) \\
\text { s.t. } \\
\alpha_{i, j} \geq 0 \forall i \forall j \\
\alpha^{\mathrm{max}} \geq \alpha_{i, j} \geq \alpha_{i, j-1} j \geq 2 \forall j \geq 2 \\
\sum_{j} P_{i, j}^{\mathrm{Gmax}}=P_{i}^{\mathrm{Gmax}} \\
\omega P_{i}^{\mathrm{Gmax}} \leq P_{i, j}^{\mathrm{Gmax}}
\end{gathered}
$$

Where: $\lambda_{n}(t)$ is the locational marginal price of node $n$ at time $t ; P_{i, j}^{\mathrm{G}}(t)$ is the bid-winning power generation of unit $i$ at the $j$ quotation stage at time $t ; \lambda_{i, j}^{G}$ is the marginal generation cost of unit $i$ at the $j$ quotation stage; $\alpha_{i, j}$ is the $j^{\prime} s$ price offer of the unit $i ; \alpha^{\max }$ is the upper limit of the quotation; $i \in \Psi_{n}$ means that unit $i$ is located on node $n ; P_{i}^{\mathrm{Gmax}}$ is the upper limit of the bidding capacity of unit $i$ in the day-ahead market; $P_{i, j}^{\mathrm{Gmax}}$ is the capacity of the $j^{\prime} s$ declaration stage of unit $i ; \omega$ is the minimum declaration ratio of the declaration stage. Formula (1) is the revenue function of the unit (minimizing the negative value of revenue); Formula (2) is the non-negative constraint on unit quotes; Formula (3) is the constraint on the upper limit of unit quotes and the monotonic increasing constraint of unit quote curves; Equation (4) is that the sum of all declared capacity of the unit is equal to the upper limit of the biddable capacity, so as to avoid the power generation companies holding capacity to raise electricity prices; formula (5) indicates that the declaration of each stage must be greater than the minimum declared proportion of biddable capacity.

\subsection{Lower model}

$$
\begin{array}{r}
\min \sum_{t} \sum_{i} \sum_{j} \alpha_{i, j} P_{i, j}^{\mathrm{G}}(t)-\sum_{t} \sum_{d} \sum_{k} \lambda_{d, k} P_{d, k}^{\mathrm{D}}(t) \\
P_{i}^{\mathrm{Gmin}} \leq \sum_{j} P_{i, j}^{\mathrm{G}}(t) \leq P_{i}^{\mathrm{Gmax}} \forall t \forall i \\
0 \leq P_{i, j}^{\mathrm{G}}(t) \leq P_{i, j}^{\mathrm{Gmax}} \forall t \forall i \\
0 \leq P_{d, k}^{\mathrm{D}}(t) \leq P_{d, k}^{\mathrm{Dmax}} \forall t \forall i \\
\sum_{d \in \Psi_{n}} \sum_{k} P_{d, k}^{\mathrm{D}}(t)=P_{n}^{\mathrm{D}}(t) \forall t \forall n
\end{array}
$$




$$
\begin{gathered}
-R_{i}^{\mathrm{D}} \leq \sum_{j} P_{i, j}^{\mathrm{G}}(t)-\sum_{j} P_{i, j}^{\mathrm{G}}(t-1) \leq R_{i}^{\mathrm{U}} \forall t \forall i \\
\sum_{i} \min \left\{R_{i}^{\mathrm{U}}, P_{i}^{\mathrm{Gmax}}-\sum_{j} P_{i, j}^{\mathrm{G}}(t)\right\} \geq S^{\mathrm{U}}(t) \\
\sum_{i} \min \left\{R_{i}^{\mathrm{D}}, \sum_{j} P_{i, j}^{\mathrm{G}}(t)-P_{i}^{\mathrm{Gmin}}\right\} \geq S^{\mathrm{D}}(t) \\
\sum_{i \in \Psi_{n}} \sum_{j} P_{i, j}^{\mathrm{G}}(t)-P_{n}^{\mathrm{D}}(t) \\
-\sum_{m \in \Theta_{n}} B_{n, m}\left(\delta_{n}(t)-\delta_{m}(t)\right)=0 \forall t \forall n \\
-P_{n, m}^{\max } \leq B_{n, m}\left(\delta_{n}(t)-\delta_{m}(t)\right) \leq P_{n, m}^{\max } \forall t \forall n \forall m \in \Theta_{n} \\
-\pi \leq \delta_{n}(t) \leq \pi \\
\delta_{n}=0, n=1 \forall t
\end{gathered}
$$

Where: $t$ is the index of the clearing time, $i$ is the index of the generator set, $j$ is the index of the quotation stage of the generator set, $d$ is the index of the demand, $k$ is the index of the demand quotation stage, $n$ and $m$ are the indexes of the nodes. $\lambda_{d, k}$ is the marginal utility of electric energy demand; $P_{d, k}^{\mathrm{D}}(t)$ is the electricity demand of demanding user $d$ in the $k$ declaration stage; $P_{i}^{\mathrm{Gmin}}$ is the minimum technical output of unit $i ; P_{n}^{\mathrm{D}}(t)$ is the load demand of node $n$ at time $t ; R_{i}^{\mathrm{D}}$ and $R_{i}^{\mathrm{U}}$ are the downward and upward climb rates of unit $i$ respectively; $S^{\mathrm{U}}(t)$ and $S^{\mathrm{D}}(t)$ are the positive and negative spinning reserve demand of the system at time $t$ respectively; $B_{n, m}$ is the line admittance of nodes $n$ to $m ; \delta_{n}(t)$ is the voltage phase angle of node $n$ at time $t ; P_{n, m}^{\max }$ is the line transmission power limit of nodes $n$ to $m$. Equation(6) is the clearing objective function for maximizing social welfare; Equation (7) is that the sum of the bid-winning power of each unit must not exceed the maximum power generation capacity and be less than the minimum technical output; Equations (8) and (9) indicate that the amount of electricity purchased shall not exceed the upper limit of declared electricity; formula (10) is that the sum of the electric quantity of all users at the same node is equal to the predicted load constraint; formula (11) is the climbing rate constraint; formula (12) and formula (13) are the spinning reserve constraint ; Equation (14) is the node power balance constraint; Equation (15) is the upper and lower limit of the branch power flow; Equation (16) is the node voltage phase angle constraint; Equation (17) indicates that the node with the network node number 1 is set as the reference node .

In the optimization model with the minimum power purchase cost as the objective function, the Lagrangian multiplier of equation (14) is the node marginal price, but in this model, the objective function of maximizing social welfare is adopted, Therefore, the node electricity price is defined as the average value of the marginal power utility increase on the power consumption side and the marginal power generation cost increase on the generation side under the unit load increment, namely:

$$
\begin{aligned}
\lambda_{n} & =\left(\partial F^{\mathrm{G}} / \partial P_{n}^{\mathrm{D}}+\partial F^{\mathrm{D}} / \partial P_{n}^{\mathrm{D}}\right) / 2 \\
F^{\mathrm{G}} & =\sum_{t} \sum_{i} \sum_{j} \alpha_{i, j} P_{i, j}^{\mathrm{G}}(t) \\
F^{\mathrm{D}} & =\sum_{t} \sum_{d} \sum_{k} \lambda_{d, k} P_{d, k}^{\mathrm{D}}(t)
\end{aligned}
$$

Where: $F^{\mathrm{G}}$ is the power generation cost on the power generation side; $F^{\mathrm{D}}$ is the power utility on the power consumption side. The settlement electricity price of formula (18) means that after the high and low matching of the declaration stages of the sender and the consumer, the average price of the last matching pair is the settlement price.

\section{Co-evolutionary algorithm solution}

For models (1)-(17), there are two classic solutions. One method replaces the lower-level model through the firstorder necessary conditions to form the original dual formula constraint, thereby transforming the bi-level model into a single-layer model. Since the feasible region of the lower-level model is a convex set, the first-order necessary condition is also a sufficient condition for the global optimization. Finally, MPEC or EPEC is formed and the market equilibrium point is obtained by solving mixed integer linear programming. The other is to directly solve the equilibrium point of the market through heuristic algorithms (such as co-evolutionary algorithms). Because the heuristic algorithm surpasses the complicated mathematical derivation, and directly seeks the equilibrium point of the market through the parameter transfer of the upper and lower bi-level models, it is significantly better than the equilibrium constraint programming in the accuracy and simplicity of the solution.

\subsection{Co-evolutionary algorithm steps}

The essence of the co-evolution algorithm to solve the market equilibrium is to set up bidding declaration information for each agent through agent simulation, and to observe the nature of the market by observing the agent's bidding behaviour. The basic ideas and steps of using co-evolutionary algorithms are as follows:

Step 1: Giving basic parameters, such as typical daily load curve, unit parameters, network parameters, market rule parameters, marginal cost of power generation companies and marginal benefits of users, etc. Construct an upper and lower two-tier equilibrium model, and use marginal cost and marginal benefit as the first bid declaration information.

Step 2: Randomly generate an independent limited strategy population set $S_{i}$ for all market entities participating in market bidding. $S_{i}$ is composed by $\mathrm{N}$ strategy elements $s_{i j}$. Each strategy $s_{i j}$ includes three 
stage of quoted volume and quotes in the declared information.

Step 3: For any bidding subject $i$ 's decision in the $t$ generation, first select the strategy corresponding to the chromosome with the highest fitness searched by other populations $(-i)$ in the $t-1$ generation as a representative to form $t-1$ representative strategy set $S_{-i}^{(t-1)^{*}}, \quad S_{-i}^{(t-1)^{*}}=\left\{s_{k}^{(t-1)^{*}} \mid k=1,2, \ldots, N ; k \neq i\right\}$. The bidding function formed by strategy $s_{i j}$ corresponding to any $j$ chromosome in population $i$ and representative strategy set $S_{-i}^{(t-1)^{*}}$ of other populations is submitted to the power dispatching agency for clearing, and the profit obtained by strategy $s_{i j}$ is set as the fitness of the individual $u_{i j}\left(s_{i j}\right)$ :

$$
u_{i j}\left(s_{i j}\right)=f_{i}\left(s_{i j}, S_{-i}^{(t-1)^{*}}\right)
$$

Where: $f_{i}(S)$ represents the profit function obtained by the $i$ 's participant after the dispatch agency clears the market for the bidding strategy set $S$ of all participants.

Step 4: $i$ takes the strategy of maximum fitness value $S_{i}^{(t)^{*}}$ as the bidding strategy of the $t$ generation, and then uses the genetic operator with the elite retention mechanism to operate the chromosomes.

$$
\begin{aligned}
& S_{i}^{(t)^{*}}=\underset{\forall j}{\arg \max } f_{i}\left(s_{i j}, S_{-i}^{(t-1)^{*}}\right) \\
& S_{i}^{(t)} \Rightarrow S_{i}^{(t+1)} \\
& \text { s.t. } \quad S_{i j} \in S_{i}^{(t)}
\end{aligned}
$$

Step 5: Repeat steps 2, 3, and 4 so that population $i \in N$ can be evaluated and evolved one by one, and finally the t-generation market is cleared.

$$
\left[\boldsymbol{P}_{\boldsymbol{t}}^{\mathrm{G}}, \boldsymbol{P}_{\boldsymbol{t}}^{\mathrm{D}}, \boldsymbol{\lambda}_{\boldsymbol{t}}\right]=\arg \min F\left(s_{1}^{(t)^{*}}, s_{2}^{(t)^{*}}, \ldots, s_{n}^{(t)^{*}}\right)
$$

Where: $F(\cdot)$ is the electricity market clearing model with the maximization of social welfare as the objective function, that is, the lower model; clearing results $\boldsymbol{P}_{t}^{\mathrm{G}}$ and $\boldsymbol{P}_{\boldsymbol{t}}^{\mathrm{D}}$ are the bidding power of the supply side and the demand side in the $t$ round of bidding respectively; $\lambda_{t}$ is the node electricity price vector.

Step 6: Repeat steps 3 to 5 until the algebra $t$ reaches the value $\mathrm{R}$ set by the simulation or stops when the market converges.

\subsection{Market Nash Equilibrium Determination}

According to the classic game theory, the Nash equilibrium can be explained as: the strategy adopted by any party is the best strategy under the strategy combination for all the other parties; when all others do not change the strategy, in order to maximize their own benefits, any party They will not (or cannot) change their own strategies. At this time, the strategy combination is a
Nash equilibrium. It can be seen that $\forall i$ satisfies the following formula:

$$
f_{t}\left(s_{i}^{(t)^{*}}, S_{-i}^{(t)^{*}}\right) \geq f_{t}\left(s_{i j}, S_{-i}^{(t)^{*}}\right) \quad \forall s_{i j} \in S_{i}
$$

Neither party has the motivation to change its own strategy, because formula (4) indicates that everyone's strategy is the optimal response to other people's strategies, and unilateral changes to their own strategies will reduce their own profits, thus the Nash equilibrium of the market is reached.

It can be seen from the framework of the algorithm that any population $i$ assumes that the strategy of other populations $(-i)$ remains unchanged, and all adopts the best strategy of the previous generation, use genetic algorithm to search subspace $S_{i}$ for random optimization. If the entire ecosystem reaches convergence after several generations of evolution, that is, the strategy $s_{i}^{(t)^{*}}$ selected by any population $i$ no longer changes, and it is the best response of $S_{-i}^{(t-1)^{*}}$, which satisfies:

$$
f_{t}\left(s_{i}^{(t)^{*}}, S_{-i}^{(t-1)^{*}}\right) \geq f_{t}\left(s_{i j}, S_{-i}^{(t-1)^{*}}\right) \quad s_{i j} \in S_{i}
$$

And since the market is in a state of convergence, satisfy:

$$
S^{(t-1)^{*}}=S^{(t)^{*}}
$$

Thus for $\forall i$ :

$$
f_{t}\left(s_{i}^{(t)^{*}}, S_{-i}^{(t)^{*}}\right) \geq f_{t}\left(s_{i j}, S_{-i}^{(t)^{*}}\right) \quad s_{i j} \in S_{i}
$$

Therefore, from the principle of co-evolutionary algorithm and the algorithm framework designed for market bidding mode, co-evolutionary algorithm has the possibility of simulating the market equilibrium. It needs to be pointed out that the focus of this article is not on the existence and uniqueness of market equilibrium, but on the use of coevolutionary algorithms to solve possible market equilibrium.

\section{Examples}

The network model adopts the PJM 5-machine 5-node model, the unit parameters are shown in Table 1, and the typical 24-hour load curve is shown in Table 2. On the user side, with loads at nodes 2,3 , and 4 , the marginal utility is 1.5 yuan $/ \mathrm{kWh}$, and the three-stage quotation strategies for 3 users are $1,0.9$ and 0.8 yuan $/ \mathrm{kWh}$ and remain unchanged in each round.

Table 1. Unit parameters.

\begin{tabular}{llllll}
\hline $\begin{array}{l}\text { Unit } \\
\text { number }\end{array}$ & $\begin{array}{l}\text { Node } \\
\text { number }\end{array}$ & $\begin{array}{l}\text { capacit } \\
\text { y/MW }\end{array}$ & $\begin{array}{l}\text { Grade } \\
\text { rate } \\
\text { /(MW/1 } \\
5 \mathrm{~min})\end{array}$ & $\begin{array}{l}\text { Lower } \\
\text { limit of } \\
\text { output }\end{array}$ & $\begin{array}{l}\text { margin } \\
\text { al cost } \\
/(\text { yuan/ } \\
\mathrm{kWh})\end{array}$ \\
\hline G1 & 1 & 500 & 62.50 & 200 & 0.28 \\
G2 & 1 & 450 & 56.25 & 175 & 0.29 \\
G3 & 3 & 400 & 50.00 & 150 & 0.30 \\
G4 & 4 & 350 & 43.75 & 125 & 0.31 \\
G5 & 5 & 300 & 37.50 & 100 & 0.32 \\
\hline
\end{tabular}


Table 2. Typical load curve.

\begin{tabular}{lllllll}
\hline$t$ & 1 & 2 & 3 & 4 & 5 & 6 \\
load & 905 & 911 & 867 & 835 & 813 & 792 \\
\hline \hline$t$ & 7 & 8 & 9 & 10 & 11 & 12 \\
load & 808 & 797 & 813 & 921 & 976 & 1024 \\
\hline \hline$t$ & 13 & 14 & 15 & 16 & 17 & 18 \\
load & 970 & 992 & 1013 & 1024 & 1019 & 1024 \\
\hline \hline$t$ & 19 & 20 & 21 & 22 & 23 & 24 \\
load & 981 & 965 & 1035 & 1073 & 1057 & 981 \\
\hline
\end{tabular}

\subsection{Market game and equilibrium analysis}

As shown in Figure 1, after a fierce game, the market entered equilibrium after about 15 rounds of bidding. In terms of bidding strategy, compared with the initial bidding strategy, the units have adopted price reduction measures in multiple rounds of games. In the example settings of this article, since the supply-demand ratio is close to $2: 1$, it is in a buyer's market. Therefore, the demand side dominates. Competition on the power generation side is fierce, and more power generation can only be obtained through price reduction, and finally converges to a lower level in multiple rounds of bidding games.

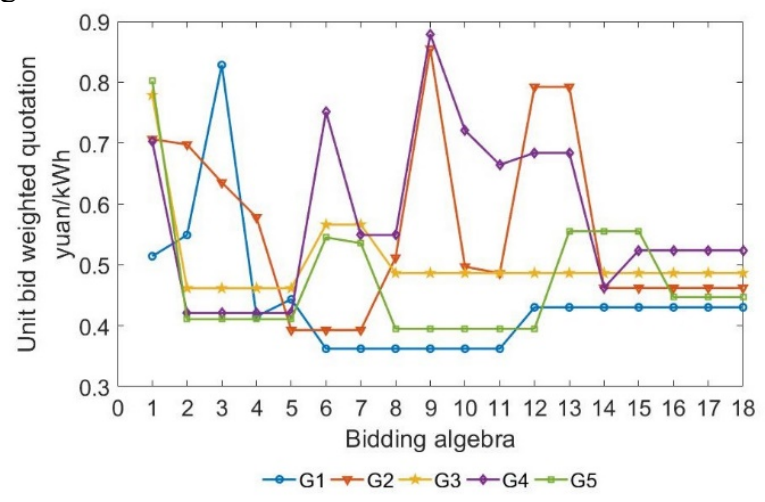

Figure 1. The process of bidding

Table 3. Comparison of Nash Equilibrium and Perfect Competition.

\begin{tabular}{llllll}
\hline \multirow{2}{*}{ unit } & \multicolumn{2}{l}{ Profit/thousand yuan } & \multicolumn{2}{l}{ Market equilibrium bidding strategy } \\
\cline { 2 - 6 } & $\begin{array}{l}\text { Game } \\
\text { equilibrium }\end{array}$ & $\begin{array}{l}\text { Perfect } \\
\text { competition }\end{array}$ & Report power/MWh & $\begin{array}{l}\text { Declare } \\
\text { price/(yuan/kWh) }\end{array}$ \\
\hline G1 & 2488.0 & 4018.4 & $(222.8,130.4,146.8)$ & $(0.311,0.327,0.703)$ \\
G2 & 1342.3 & 1617.3 & $(284, .4,108.7,56.9)$ & $(0.435,0.491,0.541)$ \\
G3 & 1312.1 & 1234.5 & $(245.7,58.5,95.8)$ & $(0.328,0.654,0.791)$ \\
G4 & 1373.6 & 799.0 & $(188.4,36.2,125.4)$ & $(0.326,0.422,0.850)$ \\
G5 & 1713.9 & 310.0 & $(215.3,45.5,39.2)$ & $(0.323,0.717,0.815)$ \\
Total profit & 8229.9 & 7979.2 & $/$ & $/$ & \\
$\begin{array}{l}\text { Market clearing } \\
\text { /(yuan/kWh) }\end{array}$ & price & 0.6629 & 0.6431 & $/$ & $/$ \\
\hline
\end{tabular}

As shown in Table 3, in the bidding strategies at the market equilibrium point, all units declare the largest proportion of bidding electricity in the first stage of the "three-stage" quotation, along with the willingness price close to the marginal generation cost. And in the second and third paragraphs, the bidding electricity will be reduced, while the bidding willingness price will be raised. Therefore, on the premise that most of its own capacity can be traded, the market's clearing electricity price should be raised as much as possible to maximize its own profits.

In the comparison between the equilibrium result of the game and the result of perfect competition, after the consideration of the game is added, the profit disparity between different units no longer changes, and smallcapacity units are not always at a disadvantage in bidding. In the case of perfect competition, all units are quoted at their marginal costs. Therefore, according to the social welfare maximization model, unit G1 has the smallest marginal cost and therefore has an absolute dominant position in the power generation market and has the highest profit. In the game equilibrium situation, although large-capacity units still maintain their advantages, their profits have dropped significantly. It can be seen that the influence of game strategy on the results of market bidding is very important. In terms of total profit, due to the influence of the market game, the unit raises the bidding price with the marginal power generation cost as the lower bound, thus raising the market clearing price, and the profit obtained by the power generation side has also been improved.

\section{Conclusion}

In this paper, the co-evolution algorithm is used to solve the upper and lower equilibrium model, and the market equilibrium result of the bidding game between power generation companies under the power spot environment is studied. The results show that under the limited strategy set, the application of co-evolution algorithm to solve the bi-level model can quickly converge to the equilibrium point of the market.

This article does not consider the impact of mediumand long-term contracts on the equilibrium of the spot market, which is also a question that needs to be studied in the next step.

\section{References}

1. Dai T, Qiao W . Optimal Bidding Strategy of a Strategic Wind Power Producer in the Short-Term Market[J] . IEEE Transactions on Sustainable Energy, 2017, 6(3): 707-719 . 
2. Guo H, Chen Q, Fang X, et al . Efficiency Loss for Variable Renewable Energy Incurred by Competition in Electricity Markets[J] . IEEE Transactions on Sustainable Energy, 2020, 11(3): 1951-1964 .

3. Yang $\mathrm{Y}$, Zhang $\mathrm{Y}$, Li F, et al .Computing All Nash Equilibria of Multiplayer Games in Electricity Markets by Solving Polynomial Equations[J] . IEEE Transactions on Power Systems, 2012, 27(1): 8191 .

4. Dai T, Qiao W . Finding Equilibria in the Pool-Based Electricity Market With Strategic Wind Power Producers and Network Constraints[J] . IEEE Transactions on Power Systems， 2016， 32(1): 389399 .

5. Ruiz C .Equilibria in an Oligopolistic Electricity Pool With Stepwise Offer Curves[J] . IEEE Transactions on Power Systems, 2012， 27(2): 752-761.

6. Wang Xian, Xie Ming, Zhang Shaohua . Analysis on equilibrium of electricity market under gridintegration of electric vehicles $[\mathrm{J}]$. Power System Technology, 2014, 31(11): 2993-2998.

7. Wang Xian, Huang Mengtao , Zhang Shaohua . Analysis of an electricity market equilibrium model with penalties for wind power's bidding deviation[J] . Power System Technology, 2016, 40(2): 602-607(in Chinese) .

8. Li R, Wei W, Mei S, et al .Participation of an Energy Hub in Electricity and Heat Distribution Markets: An MPEC Approach[J] . IEEE Transactions on Smart Grid, 2019, 10(4): 3641-3653 .

9. Wang $\mathrm{C}$, Wei W, Wang J, et al . Strategic Offering and Equilibrium in Coupled Gas and Electricity Markets[J] .IEEE Transactions on Mobarakeh A S ,

Mohsenian-Rad H . Optimal Bidding in PerformanceBased Regulation Markets: An MPEC Analysis With System Dynamics $[\mathrm{J}]$. IEEE Transactions on Power Systems, 2017, 32(2): 1282-1292 .

10. Ameri M, Rahimiyan M, Latify M A . Capacity Withholding Constrained by Operational Limits of Generation under Financial Virtual Divestiture in a Day-Ahead Market[J] . IEEE Transactions on Power Systems, 2017, 33(1): 771-780

11. AN Xuena, ZHANG Shaohua, LI Xue .Equilibrium analysis of oligopolistic electricity markets considering tradable green certificates [J] .Automation of Electric Power Systems, 2017, 41 (9) : 84-89 .

12. Xie Chang, Wang Beibei, Zhao Shengnan, et al . Equilibrium solution for electricity market based on bi-level particle swarm optimization algorithm[J] .Power System Technology, 2018, 42(4): 1170-1176(in Chinese) .
13. Liu Yumeng, Chen Haoyong, Huang Long, et al . Equilibrium model of electricity market based on multi-swarm co-evolution[J] . Power System Protection and Control, 2020, 48(10): 38-45 .

14. Zhang Hongli, Li Yuanmei . Reactive power optimization based on filter hybrid coevolution algorithm[J] . Control and Decision, 2017, 32(9): 1701-1706 .

15. WEN Xu, GUO Lin, YAN Wei, et al . A reactive optimization model with flexible objectives for power systems containing voltage-violated buses [J] .Proceedings of the CSEE, 2017, 37(6): 16761685

16. Li Xiaozhu, Wang Weiqing, Wang Haiyun, et al . Robust Optimized Operation Strategy for Crossregion Flexibility with Bilateral Uncertainty of Load Source[J] High Voltage Engineering, 2020, 46(05): 1538-1549 .

17. Yang Yan, Zhang Yao, Chen Haoyong, et al . An electricity market equilibrium model considering uncertainty in power system operation[J] . Power System Technology, 2012, 36(7): 100-105 .

18. Yang Yan, Chen Haoyong, Zhang Yao, et al . A coevolutionary approach to calculate equilibrium for oligopolistic electricity market[J] . Automation of Electric Power Systems, 2009， 33(18)：42-46+115 (in Chinese) .

19. SHI Ke, CHEN Haoyong, LI Peng, et al . Analysis on two kinds of electricity market clearance mechanism based on co-evolution[J] . Automation of Electric Power Systems, 2019, 43(9): 68-77.

20. Mobarakeh A S , Mohsenian-Rad H . Optimal Bidding in Performance-Based Regulation Markets: An MPEC Analysis With System Dynamics[J] . IEEE Transactions on Power Systems, 2017, 32(2): 12821292 . 